\title{
Cloning of 3-Hydroxy-3-methylglutaryl-coenzyme A Reductase Gene from Vanda Mimi Palmer and Its Heterologous Expression in Escherichia coli
}

\author{
Seow Ling Teh ${ }^{1}$, Janna Ong Abdullah ${ }^{1}$, Parameswari Namasivayam ${ }^{2} \&$ Gwendoline Cheng Lian Ee $^{3}$ \\ ${ }^{1}$ Department of Microbiology, Faculty of Biotechnology and Biomolecular Sciences, Universiti Putra Malaysia, \\ Selangor Darul Ehsan, Malaysia \\ ${ }^{2}$ Department of Cell and Molecular Biology, Faculty of Biotechnology and Biomolecular Sciences, Universiti \\ Putra Malaysia, Selangor Darul Ehsan, Malaysia \\ ${ }^{3}$ Department of Chemistry, Faculty of Science, Universiti Putra Malaysia, Selangor Darul Ehsan, Malaysia \\ Correspondence: Janna Ong Abdullah, Department of Microbiology, Faculty of Biotechnology and Biomolecular \\ Sciences, Universiti Putra Malaysia, UPM 43400 Serdang, Selangor Darul Ehsan, Malaysia. Tel: \\ 60-389-466-697. E-mail: janna@biotech.upm.edu.my
}

Received: May 9, 2012 Accepted: June 14, 2012 Online Published: August 15, 2012

doi:10.5539/jps.v1n2p140 URL: http://dx.doi.org/10.5539/jps.v1n2p140

\begin{abstract}
Plant 3-hydroxy-3-methylglutaryl-CoA-reductase (HMGR) is involved in the conversion of HMG-CoA into mevalonate (MVA), which yields a biologically active isoprenoid precursor, isopentenyl pyrophosphate (IPP) unit. To date, heterologous expression of HMGR isolated from orchidaceae family has not been reported. The aims of this study were to isolate, clone, over-express and functionally characterize the cDNA encoding a 3-hydroxy-3-methylglutaryl-CoA-reductase of Vanda Mimi Palmer (VMPHMGR) in Escherichia coli strain BL21 (DE3) pLysS. The deduced VMPHMGR contains an open reading frame (ORF) of 1689 bp and generates a protein of 562 amino acids with a calculated molecular mass of $59780 \mathrm{Da}$ and a predicted pI value of 6.63, which is $76 \%$ identical to other plant HMGRs. Expression analysis of VMPHMGR transcript by real-time RT-PCR showed that it was differentially regulated. Primers with appropriate restriction sites were used to amplify and facilitate in-frame cloning of the VMPHMGR into pET32(a). The expression of VMPHMGR, in E. coli, fused to N-terminal thioredoxin (Trx·Tag), S·Tag and His·Tag fusion proteins in pET32(a) yielded a partially soluble recombinant protein. This expressed VMPHMGR was subjected to functional enzymatic assay and GCMS analysis of the end products detected dehydromevalonic lactone and pantolactone, which were derivatives of mevalonate lactone.
\end{abstract}

Keywords: isoprenoid, mevalonate pathway, Vanda Mimi Palmer, 3-hydroxy-3-methylglutaryl-coenzyme A reductase, vandaceous orchid

\section{Introduction}

Plants have the capability to synthesize many volatile metabolites, either primary or secondary, with varied functions (Goff \& Klee, 2006). Floral scent has a significant impact in plant reproduction as it attracts a variety of animal pollinators, especially insects. Most volatile compounds are derived from the three major biosynthesis pathways: phenylpropanoids, fatty acid derivatives and terpenoids (Pichersky, Noel, \& Durareva, 2006). In plants, two distinct pathways, the methyl- ${ }_{\mathrm{D}}$-erythritol 4-phosphate (MEP) and mevalonate (MVA) pathways are involved in the biosynthesis of isoprenoid compounds (Rohmer, Knani, Simonin, Sutter, \& Sahm, 1993).

Detailed chemical analysis of volatile compositions and the identification of secondary metabolites related to scent production have led to the discovery of a number of novel flower scent-related genes including 3-hydroxy-3-methylglutaryl-coenzyme A reductase [HMGR] (Guterman et al., 2002; Knudsen \& Gershenzon, 2006; Mohd-Hairul, Namasivayam, Gwendoline, \& Janna, 2010) and terpene synthase (Dudavera et al., 2003). HMGR catalyzes the conversion of HMG-CoA into mevalonate (MVA) to yield a biologically active isoprenoid precursor, isopentenyl pyrophosphate (IPP) unit (Friesen \& Rodwell, 2004; Jiang et al., 2006; Wang et al., 2007), which serves as the basis for the biosynthesis of molecules used in many metabolic processes such as steroid biosynthesis (Pitera, Paddon, Newman, \& Keasling, 2007), terpenoid biosynthesis (Cheng et al., 2007) and 
carotenoid biosynthesis (Tada et al., 1990). The isolation and molecular characterization of plant HMGRs have been performed in both monocots and dicots such as Arabidopsis thaliana (Dudareva et al., 2005), rice (Ha, Lee, Kim, \& Hwang, 2001), Camptotheca acuminate (Maldonado-Mendoza, Vincent, \& Nessler, 1997), Ganoderma lucidum (Shang et al., 2008), and hazel (Wang et al., 2007).

So far, HMGR gene has not been reported from vandaceous orchids or any orchid genera. Orchidaceae is one of the largest flowering plant families and evolutionary-wise, they are highly complex with varied floral structures. $H M G R$ information gathered from this highly complex plant would enhance our understanding of this gene and its product, which is involved in the first rate-limiting step for isoprenoid biosynthesis. Therefore, in this paper we report our attempt in the isolation, cloning and functional characterization of the VMPHMGR protein in Escherichia coli. In our previous study, a Vanda Mimi Palmer expressed sequence tag database (VMPEST) (Teh, Chan, Janna, \& Namasivayam, 2011) was generated to facilitate the identification of potential fragrance-related genes in this vandaceous orchid. From the VMPEST, a clone bearing high sequence homology to published HMGR was selected for this study. This finding of HMGR in orchid could provide an alternative resource for the enzyme and this might also increase the value of orchids besides being regarded as ornamental plants only. On top of that, it may prove interesting to elucidate potential compounds that might have synergetic effects with this enzyme in vivo.

\section{Material and Methods}

\subsection{Sample Collection}

Vanda Mimi Palmer potted plants were bought and maintained at the United Orchid Plantation nursery, Rawang, Selangor Darul Ehsan, Malaysia, under a 20-30\% shade with temperature ranging between $25-30^{\circ} \mathrm{C}$. The plants were brought and placed outside the laboratory building, which has almost similar conditions as the nursery, prior to samples collection (Chan, Janna, Namasivayam, \& Maziah, 2009; Mohd-Hairul, 2010). The VMP floral samples for the RNA work were harvested according to their diameters: bud $(0.8-1.4 \mathrm{~cm})$, blooming $(3.0-5.0 \mathrm{~cm})$ and full-bloom $(5.0-7.0 \mathrm{~cm})$ as described by Chan et al. (2011). Various tissues (sepals, petals, lips, stems, stalks, columns, roots and leaves) were also collected and stored at $-80^{\circ} \mathrm{C}$ until ready to be used for total RNA extraction.

\subsection{Total RNA Extraction}

The mortar, pestle and spatula used in the total RNA extraction were baked at $200^{\circ} \mathrm{C}$ for 2 to 3 hours prior to use to ensure removal of contaminating RNase. Total RNA were extracted from all frozen floral tissues, as mentioned earlier, using the standard CTAB protocols of Yu and Goh (2000).

\subsection{Full Length cDNA Isolation of VMPHMGR from Vanda Mimi Palmer}

A partial cDNA sequence that encodes a putative 3-hydroxy-3-methylglutaryl-CoA-reductase of Vanda Mimi Palmer (VMPHMGR) (GenBank accession no. GW392501) was selected from a Vanda Mimi Palmer floral EST database (Teh et al., 2011). This cDNA transcript lacks the 5'-region. The RACE cDNA Amplification Kit (Clontech, CA, USA) and Advantage 2 Polymerase Mix (Clontech, CA, USA) were used according to the manufacturers' instructions to isolate the full $V M P H M G R$ cDNA sequence. A gene specific primer (5'-GGATTTCCCTCTTCCCTCGATCCAA-3'), and a universal primer mix (UPM) made up of a long primer: 5'-CTAATACGACTCACTATAGGGCAAGCAGTGGTATCAACGCAGAGT-3' and a short primer: 5'-CTAATACGACTCACTATAGGGC- 3', were used to isolate the 5'-end of the VMPHMGR transcript with a PCR cycling conditions of denaturation at $94^{\circ} \mathrm{C}$ for 30 seconds, annealing at $65^{\circ} \mathrm{C}$ for 30 seconds and extension at $72^{\circ} \mathrm{C}$ for 3 minutes, for a total of 25 cycles using a gradient thermal cycler (Eppendorf, Hamburg, Germany). The RACE PCR product was subsequently gel purified using a gel extraction kit (Qiagen, CA, USA) and cloned into the yT \& A cloning vector (Yeastern Biotech, Taipei, Taiwan) prior to sequencing (Bioneer, Daejeon, Korea) The sequence obtained was analysed using the BLASTN, BLASTX, CAP CONTIG, Biology WorkBench 3.2 (http://workbench.sdsc.edu/), BioEdit version 7.0.9 software and ClustalW.

In order to obtain the full $V M P H M G R$ transcript sequence, a pair of gene specific primer was designed at the 5 '-end of the forward (5'- GGGCAAGCAGTGGTATCAACGCAGA-3') and reverse (5'-GTCATCAAGTCGAAGCAGCTTTGGTC-3') primers. The PCR reaction was pre-denatured at $94^{\circ} \mathrm{C}$ for 3 minutes, denatured at $94^{\circ} \mathrm{C}$ for 45 seconds, annealed at $65^{\circ} \mathrm{C}$ for 40 seconds, extended at $72^{\circ} \mathrm{C}$ for 90 seconds for a total of 35 cycles and a final extension at $72^{\circ} \mathrm{C}$ for 10 minutes. The PCR product was then purified, cloned into the yT\&A vector (Yeastern Biotech, Taipei, Taiwan), and then transformed into competent E. coli strain DH5 $\alpha$ cells. Plasmid DNA was isolated from the transformed cells and sequenced. Sequence analysis was performed using the BLASTX, ClustalW (http:/www.ebi.ac.uk/Tools/clustalw2/index.html), BioEdit version 7.0.9 (Tom 
Hall, Ibis Biosciences 1999), WoLF PSORT (http://wolfpsort.org/), iPSORT (http://ipsort.hgc.jp/) and ExPASy (http://www.expasy.ch/tools/). MEGA 4.0 (http://www.megasoftware.net/) was used to infer the phylogenetic tree and estimate the relationship between the putative VMPHMGR and other plants' HMGRs.

\subsection{Real-time Quantitative RT-PCR}

The first-strand cDNA used in the quantitative real-time RT-PCR reaction was synthesized using a reverse transcription kit (Qiagen, CA, USA). Verification of differentially expressed genes was performed using a Bio-Rad iCycler (iCycler iQ5 Multicolor Real-Time PCR Detection System) and 2x SYBR Green I Hot-Start real-time PCR-Mix (GeneCraft, Cologne, Germany). Each cDNA sample was diluted 10 folds prior to use in the real-time quantitative RT-PCR reaction. The thermal cycling conditions were as follows: activation of DNA polymerase at $95^{\circ} \mathrm{C}$ for 1 minute, followed by 40 cycles of amplification at $95^{\circ} \mathrm{C}$ for 10 seconds, $59^{\circ} \mathrm{C}$ for 20 seconds, $72^{\circ} \mathrm{C}$ for 20 seconds. Four technical replicates were carried out for the reaction of each biological replicate. A master mix without the cDNA template was used as a negative control in the reaction. The relative gene expression level was calculated using the geometric mean method of Vandesompele et al. (2002) with actin (GenBank accession no. AF246716), cyclophilin (GenBank accession no. GW393531) and tubulin (GenBank accession no. GW393564) as reference transcripts (as described by Mohd-Hairul, 2010) for comparison of the VMPHMGR expression levels at different time points (2-hour intervals within a 24-hour cycle), at different developmental stages (buds, blooming and full-bloom flower), and in different types of tissues/organs (sepals, petals, lips, stems, stalks, columns, roots and leaves). The details of the primers used in the real-time RT-PCR experiment are shown in Table 1. Statistical significance of the relative expression levels of each gene transcript was assessed using an analysis of variance (ANOVA) at $p<0.05$ (Karlen, McNair, Perseguers, Mazza, \& Mermod, 2007).

Table 1. Primers used in real-time RT-PCR

\begin{tabular}{ccl}
\hline $\begin{array}{c}\text { Primer identity } \\
\text { (amplicon size) }\end{array}$ & $\begin{array}{c}\text { GenBank } \\
\text { accession }\end{array}$ & \multicolumn{1}{c}{ Primer sequence } \\
\hline Actin (236bp) & AF246716 & $\begin{array}{l}\text { Forward: 5'-CAGTGTTTGGATTGGAGGTTC-3' } \\
\text { Reverse: 5'-CCAGCAGCAGTCAGGAAAA-3' }\end{array}$ \\
Tubulin (227bp) & GW393564 & $\begin{array}{l}\text { Forward: 5'-CTCCCGCATTGACCATAAAT-3' } \\
\text { Reverse: 5'-GGAACCACACCCAAACTCTC-3' }\end{array}$ \\
Cyclophilin (200bp) & GW393531 & $\begin{array}{l}\text { Forward: 5'-TTGGATGTCGTGAAGGCAAT-3' } \\
\text { Reverse: 5'-CAACACAAGAAGATAGCACAGCA-3' } \\
\text { Putative VMPHMGR (182bp) }\end{array}$ \\
& GW392501 & $\begin{array}{l}\text { Forward: 5'-GAAAGGCGCAAACATGGAGTCT-3' } \\
\text { Reverse: 5'-CAAGTGGAAGCAGCTTTGGTCA-3' }\end{array}$ \\
\hline
\end{tabular}

Identities of the primers designed for $V M P H M G R$ and the endogenous controls used in the real-time quantitative RT-PCR experiments are summarised in Table 1. The amplicon size for each primer set is indicated in parentheses.

\subsection{Heterologous Expression of VMPHMGR in Escherichia coli}

A mitochondrial targeting peptide was found in VMPHMGR using the iPSORT and SignalP 3.0 programme (Bendsten, Nielsen, Heijne, \& Brunak, 2004). Hence, the first 30 amino acids were excluded in the cloning of VMPHMGR into the pET32(a) vector for expression analysis in E. coli. This contributed to a recombinant truncated protein with a calculated molecular weight of $70570 \mathrm{Da}$ [including the Trx $\cdot \mathrm{Tag}, \mathrm{S} \cdot \mathrm{Tag}$ and His·Tag fusion proteins at the N-terminal]. The truncated $V M P H M G R$ cDNA fragment was amplified with a forward primer: 5'-TAGAATTCTTCCGCCGGCCGCAT-3' and reverse primer: 5'GATCTCGAGTCAAGTGGAAGCAGC-3', with EcoRI and XhoI restriction sites (underlined) engineered at the 5 ' end of the forward and reverse primers, respectively. The PCR amplification was carried out in a gradient thermal cycler (Eppendorf, Hamburg, Germany) using the KOD Hot Start DNA polymerase (Novagen, Darmstadt, Germany). The reaction mixture was first heated at $95^{\circ} \mathrm{C}$ for 2 minutes, amplified for 35 cycles at $95^{\circ} \mathrm{C}$ for 30 seconds with an annealing temperature of $65^{\circ} \mathrm{C}$ for 30 seconds and an extension at $72^{\circ} \mathrm{C}$ for 90 seconds. This was followed by a final extension at $72^{\circ} \mathrm{C}$ for 10 minutes. The PCR product was then digested with 
EcoRI and $X h o I$, purified and ligated into the pET32(a) vector. Hereafter, the construct was designated as pET32(a)::VMPHMGR and was verified by colony PCR, restriction enzyme digestion and sequencing prior to heterologous expression. The transformation of pET32(a)::VMPHMGR construct into E. coli strain BL21(DE3) pLysS was carried out following the protocol provided in the Novagen BL21(DE3) pLysS kit (Merck, Darmstadt, Germany).

\subsection{Recombinant Protein VMPHMGR Expression and Purification}

Two millilitres of an overnight culture of $E$. coli harbouring the pET32(a)::VMPHMGR were inoculated into 50 $\mathrm{ml}$ of fresh Luria Bertani (LB) broth supplemented with $34 \mu \mathrm{g} / \mathrm{ml}$ chloramphenicol and $50 \mu \mathrm{g} / \mathrm{ml}$ ampicillin. The culture was grown with shaking at $200 \mathrm{rpm}$ and $37^{\circ} \mathrm{C}$ to an $\mathrm{OD}_{600}$ of approximately $0.5-0.7$ prior to induction with $0.5 \mathrm{mM}$ isopropyl- $\beta$-D-thiogalacto-pyranoside (IPTG). This was followed by further incubation for 4 hours at $30^{\circ} \mathrm{C}$ with shaking at $220 \mathrm{rpm}$.

The cell culture was harvested by centrifugation at $4000 \mathrm{x} g$ for 15 minutes at $4^{\circ} \mathrm{C}$. Extraction of the targeted protein was carried out using the BugBuster Protein Extraction Reagent (Merck, Darmstadt, Germany). The wet weight of the bacterial cells was obtained and the BugBuster Reagent was added per gram of bacterial cells. Approximately $0.5 \mu \mathrm{l}$ of Benzonase (Merck, Darmstadt, Germany) was added and the cells mixture was incubated at room temperature on a shaking platform at $60 \mathrm{rpm}$ for 20 minutes. The insoluble proteins and cell fragments were precipitated by centrifugation at $8000 \mathrm{xg}$ for 20 minutes at $4^{\circ} \mathrm{C}$ while the supernatant (soluble proteins) was further centrifuged at $8000 \mathrm{x} g$ for 10 minutes at $4^{\circ} \mathrm{C}$. The soluble crude VMPHMGR sample was concentrated by centrifugation at 10,000 x $g$ for 45 minutes at $4^{\circ} \mathrm{C}$ using an Amicon Ultra- 4 centrifugal filter unit of a $30 \mathrm{kDa}$ protein exclusion size (Millipore, TechLink, Singapore).

Subsequently, the recombinant VMPHMGR protein was purified using the His SpinTrap kit (GE Healthcare, Uppsala, Sweden). The column was equilibrated with five column volumes (5 CVs, $3 \mathrm{ml}$ in total) of binding buffer (1X phosphate buffer stock solution and $20 \mathrm{mM}$ imidazole, $\mathrm{pH}$ 7.4). Approximately $600 \mu \mathrm{l}(1 \mathrm{CV})$ of binding buffer were added, followed by centrifugation for 1 minute at $1000 \mathrm{xg}$ at $4^{\circ} \mathrm{C}$. After that, $600 \mu \mathrm{l}$ of the crude protein was added (each application) and centrifuged. The bound protein was washed with $10 \mathrm{CVs}$ of the binding buffer, followed by elution twice with the elution buffer $(100 \mu$ leach of $1 \mathrm{X}$ phosphate buffer and 200 $\mathrm{mM}$ imidazole). Step-wise purification was performed on the induced soluble fraction of VMPHMGR with imidazole at 100,200,300, 400 and $500 \mathrm{mM}$. The mixture of proteins was separated based on their molecular weights using a $0.75-\mathrm{mm}$ thickness sodium dodecyl sulphate-polyacrylamide gel. The detection and verification on the expressed recombinant HMGR protein was carried out using the his-tag monoclonal antibody and HRP Chemiluminescent Detection kit (Amresco, OH, USA).

\subsection{Functional Enzymatic Assay}

Gas chromatography-mass spectrophotometry (GC-MS) was applied to detect the enzymatic end products from the HMGR samples (crude induced protein, crude un-induced protein, and his-tag purified protein) and crude extract from BL21(DE3)pLysS:pET32(a) [without insert, as a negative control]. The enzymatic assay was carried out using the protocols adapted from Chappell, Wolf, Proulx, Ceullar, and Saunders (1995) and Pitera et al. (2007) with some modifications. A GC glass vial containing $4 \mathrm{mg}$ of protein, $100 \mathrm{mM} \mathrm{KH}{ }_{2} \mathrm{PO}_{4}(\mathrm{pH} 7.0), 3$ mM NADPH (Merck, Darmstadt, Germany) and $20 \mu \mathrm{M}$ HMG-CoA (Fluka, MO, USA) was incubated at $30^{\circ} \mathrm{C}$ with gentle shaking at $220 \mathrm{rpm}$ for 2 hours. The reaction mixture was then added with $500 \mathrm{mM} \mathrm{HCl}$ to convert the mevalonate produced to mevalonic acid lactone, followed by an additional incubation at $30^{\circ} \mathrm{C}$ for 15 minutes with shaking at $220 \mathrm{rpm}$. An equal amount of pentane was then added to the vial and vortexed for 5 minutes. Finally, the pentane extract was transferred to a fresh GC glass vial before analysis. The pentane extract of each sample was analyzed using the GCMS-QP2010 Plus system (Shimadzu, Tokyo, Japan). The GC column used was a fused silica capillary column (SGE BPX-5, $30 \mathrm{M} \times 0.25 \mathrm{~mm} \times 0.25 \mu \mathrm{m})$ with helium $(53.6 \mathrm{kPa})$ as a carrier gas at a constant flow rate of $1 \mathrm{ml}$ per min. A splitless injection mode with an injection volume of $0.1 \mu 1$ was used. The GC column oven temperature profiles were as follows: 3 minutes at $50^{\circ} \mathrm{C}$, followed by increments of $10^{\circ} \mathrm{C}$ /minute to $250^{\circ} \mathrm{C}$, and a final temperature at $250^{\circ} \mathrm{C}$ for 10 minutes. The scan range of the mass spectrum was recorded for the $\mathrm{m} / \mathrm{z}$ values of 50 to 450 . The retention time and mass spectrum of mevalonate lactone (as standard) were obtained by comparing with the National Institute of Standards and Technology (NIST 2008) and Wiley mass spectral libraries (Scientific Instrument Services, NJ, USA). The single-ion peaks generated from each protein sample were integrated and compared with the peaks generated using the standard, mevalonate lactone (Sigma-Aldrich, Steinheim, Germany). 


\section{Results and Discussion}

\subsection{Sequence Analysis of Full Length VMPHGMR cDNA Transcript}

The full length cDNA of VMPHMGR comprises of 1920 bp with an open reading frame (ORF) of $1689 \mathrm{bp}$ encoding a 562 amino acids protein. It is flanked by 123 bp of $5^{\prime}$-untranslated region and 108 bp of 3 '-untranslated region including a poly-A tail. The deduced protein has a calculated molecular weight of 59780 $\mathrm{Da}$ (BioEdit version 7.0.9) and a predicted isoelectric point (pI) of 6.63 (http://ipsort.hgc.jp/). This sequence was submitted to the GenBank with the accession number JF519820. VMPHMGR is predicted to have a mitochondrial targeting peptide for the first 30 amino acids using the iPSORT and SignalP 3.0 programme (Bendsten et al., 2004). We consequently excluded the first 30 amino acids in the cloning of VMPHMGR into the pET32(a) for expression analysis in E. coli as described in this paper. Studies by other researchers have demonstrated that deletion of the N-terminal domain of HMGR still produced an active protein. Truncation of the N-terminal transmembrane domain of Saccharomyces cerevisiae Hmglp was found to produce an active cytosolic truncated protein (Donald, Hampton, \& Fritz, 1997; Jackson, Hart-Wells, \& Matsuda, 2003). When this truncated gene was expressed in Neurospora crassa, it was found to be functional (Wang \& Keasling, 2002).

The Blast Search and multiple sequence alignment analysis with CLUSTAL W (http://align.genome.jp/) revealed that VMPHMGR was identical to the HMGR of many other plant species, such as Oryza sativa Indica (76\%; GenBank accession no. Q9XHL5), Zea mays (75\%; GenBank accession no. CAA70440), Morus alba (74\%; GenBank accession no. AAD03789.1), Ricinus communis (73\%; GenBank accession no. EEF52919.1) and Salvia miltiorrhiza (72\%; GenBank accession no. ACD37361), suggesting that VMPHMGR belongs to the HMGR family. The N-terminal end of VMPHMGR was quite distinct in length and amino acid composition while the C-terminal catalytic domain was highly identical to other plant species (Figure 1). Kevei et al. (2007) reported that the availability of this catalytic motif aids the NADPH-dependent reduction of HMG-CoA to mevalonate.

Phylogenetic analysis suggested a divergence of the known HMGRs into two classes: Class I enzymes of eukaryotes and some archaea, and Class II enzymes of prokaryotes (Friesen \& Rodwell, 2004). The structural comparison between both classes was earlier reviewed by Istvan (2001). The catalytic domains among the eukaryotes are said to be highly conserved. VMPHMGR has two conserved HMG-CoA binding motifs [EMPVGYVQLP $\left(\mathrm{aa}_{232}-\mathrm{aa}_{241}\right)$ and TTEGCLVA $\left(\mathrm{aa}_{261}-\mathrm{aa}_{268}\right)$ ] and two NADPH binding motifs [DAMGMNM $\left(\mathrm{aa}_{357}-\mathrm{aa}_{363}\right)$ and GTVGGGT $\left(\mathrm{aa}_{506}-\mathrm{aa}_{512}\right)$ ] (Figure 1). These motifs are also found in other plants' HMGRs (Jiang et al., 2006; Wang et al., 2007). 

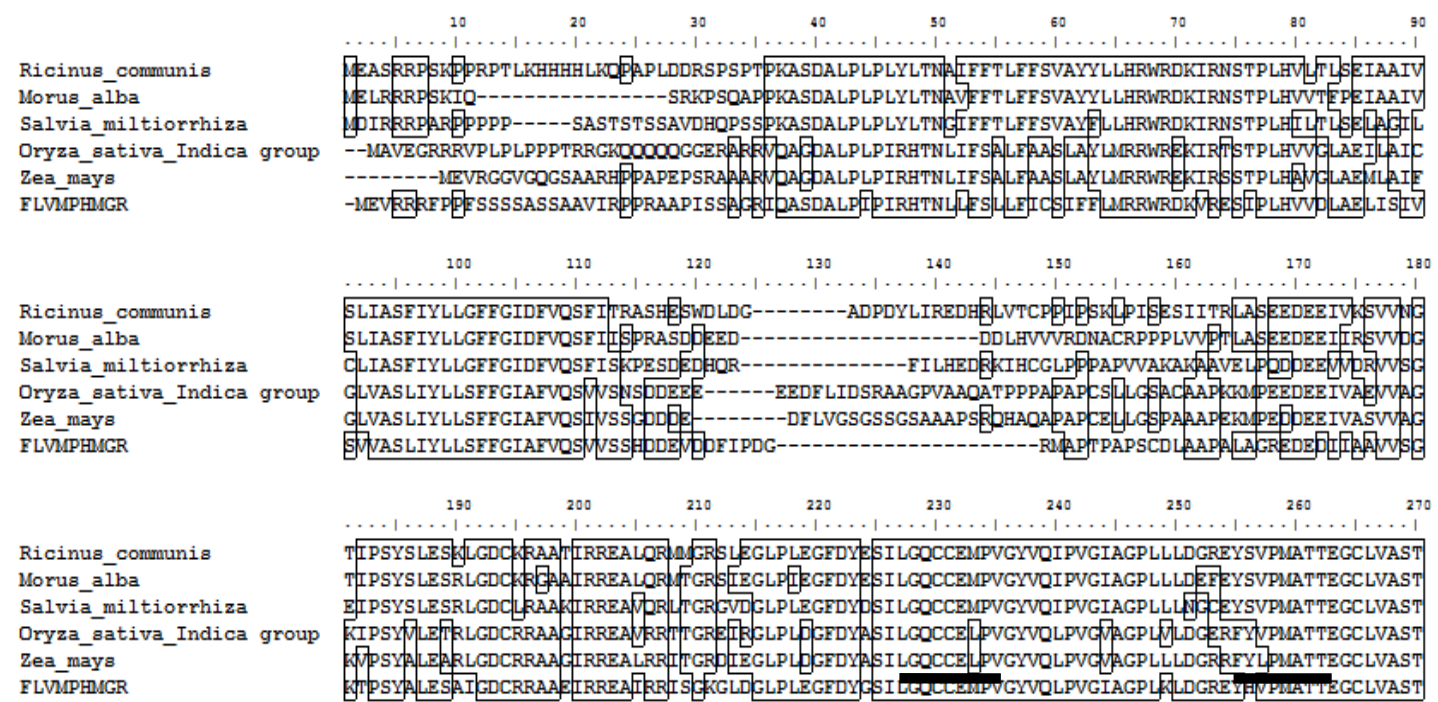

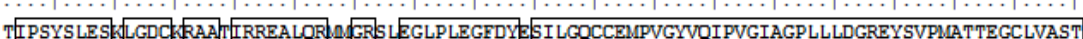

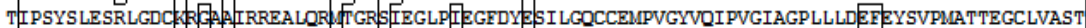

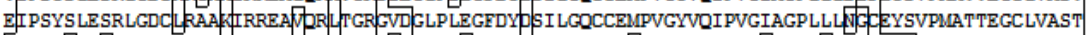

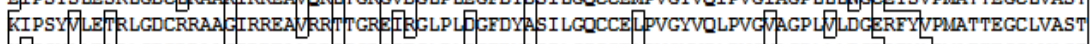

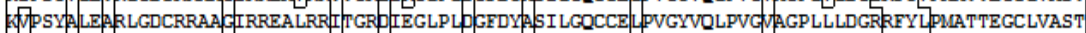

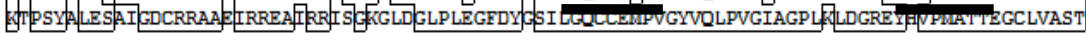

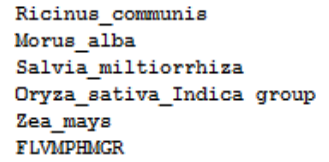

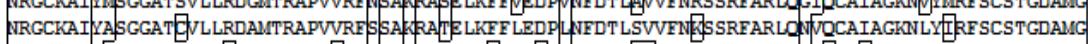

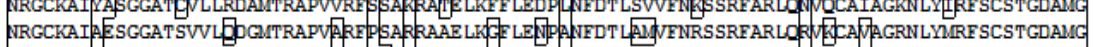

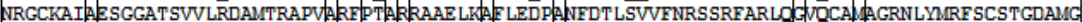

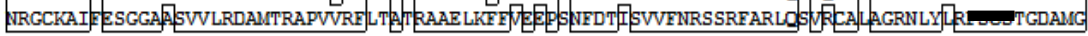

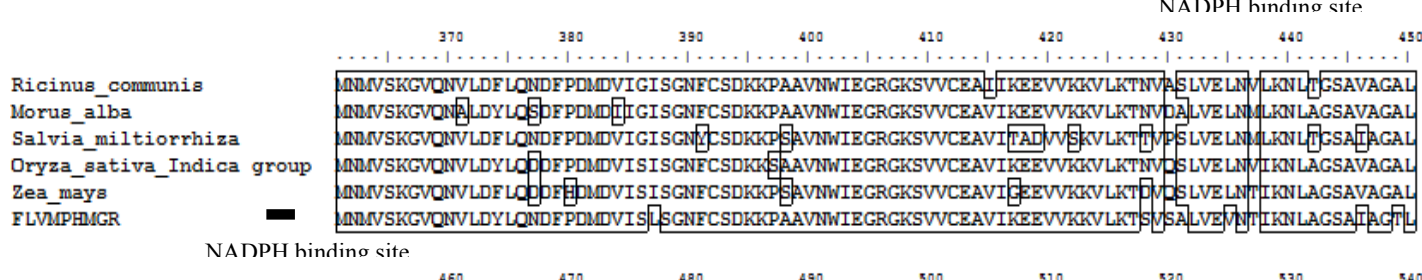

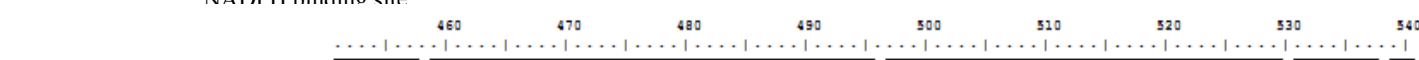

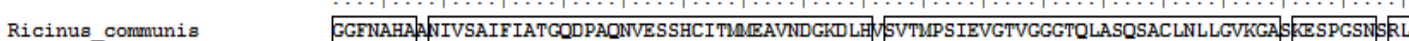

Morus_alba Salvia_miltiorrhiza

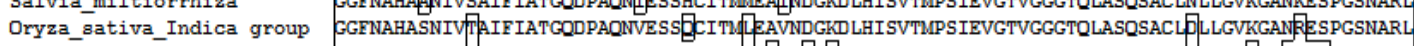

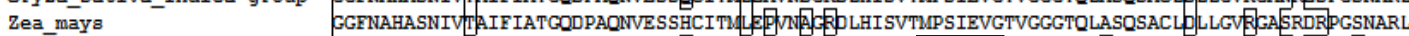
FLVMPHMGR GGFNAHASNIVSAIFIATGQDPAQNVESSPCITMLEAVNDGKDLHISVTVIPSIEVGTVGGGTQLTSQSACLNLLGVKGANYESPGTINARL

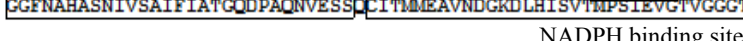

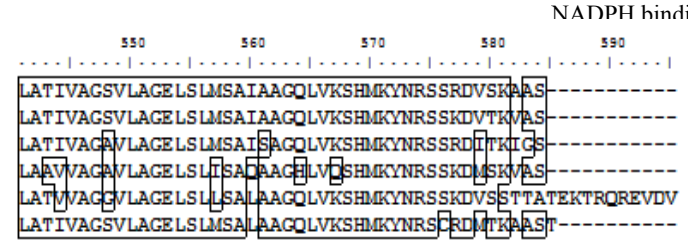

Figure 1. Multiple alignment of the deduced amino acid sequence of VMPHMGR (putative 3-hydroxy-3-methylglutaryl-CoA-reductase) and other closely related protein sequences from Ricinus communis (GenBank accession no. EEF52919.1), Morus alba (GenBank accession no. AAD03789.1), Salvia miltiorrhiza (GenBank accession no. ACD37361), Oryza sativa Indica group (GenBank accession no. Q9XHL5) and Zea mays (GenBank accession no. CAA70440).

The HMG-CoA binding sites and NADPH binding motifs are underlined

From the TMHMM 2.0 (http://www.cbs.dtu.dk/services/TMHMM/) prediction analysis, two trans-membrane regions were found in the polypeptide chain of VMPHMGR [one was located between $\operatorname{Pro}_{42}(\mathrm{P})$ and $\mathrm{Met}_{64}(\mathrm{M})$, while the other was positioned between $\mathrm{Leu}_{85}(\mathrm{~L})$ and $\left.\mathrm{Val}_{107}(\mathrm{~V})\right]$. Secondary structure analysis using SOPMA (Geourjon \& Deleage, 1995) shows that the putative VMPHMGR peptide is rich in alpha helices (46.98\%) and random coils $(36.30 \%)$ while extended strands $(12.10 \%)$ and beta turns $(4.63 \%)$ are distributed intermittently. These structural elements patterns are similar to the HMGR reported previously (Jiang et al., 2006).

From the phylogenetic tree constructed (Figure 2) to compare VMPHMGR evolutionary relationship with other 
reported plant HMGRs, it seems that all the plants $H M G R s$ share a common ancestry and VMPHMGR is clustered closely to the HMGR from Oryza sativa. This is not surprising considering that both are monocot plants. Although Zea mays shows a common conserved catalytic region, it deviates at the $\mathrm{N}$ terminus [the membrane spanning region] (Nelson, Doerner, Zhu, \& Lamb, 1994) and the C terminus (Figure 1). The divergence is obvious between Zea mays and VMPHMGR whereby VMPHMGR sequence contains several gaps at the $\mathrm{N}$ terminus.

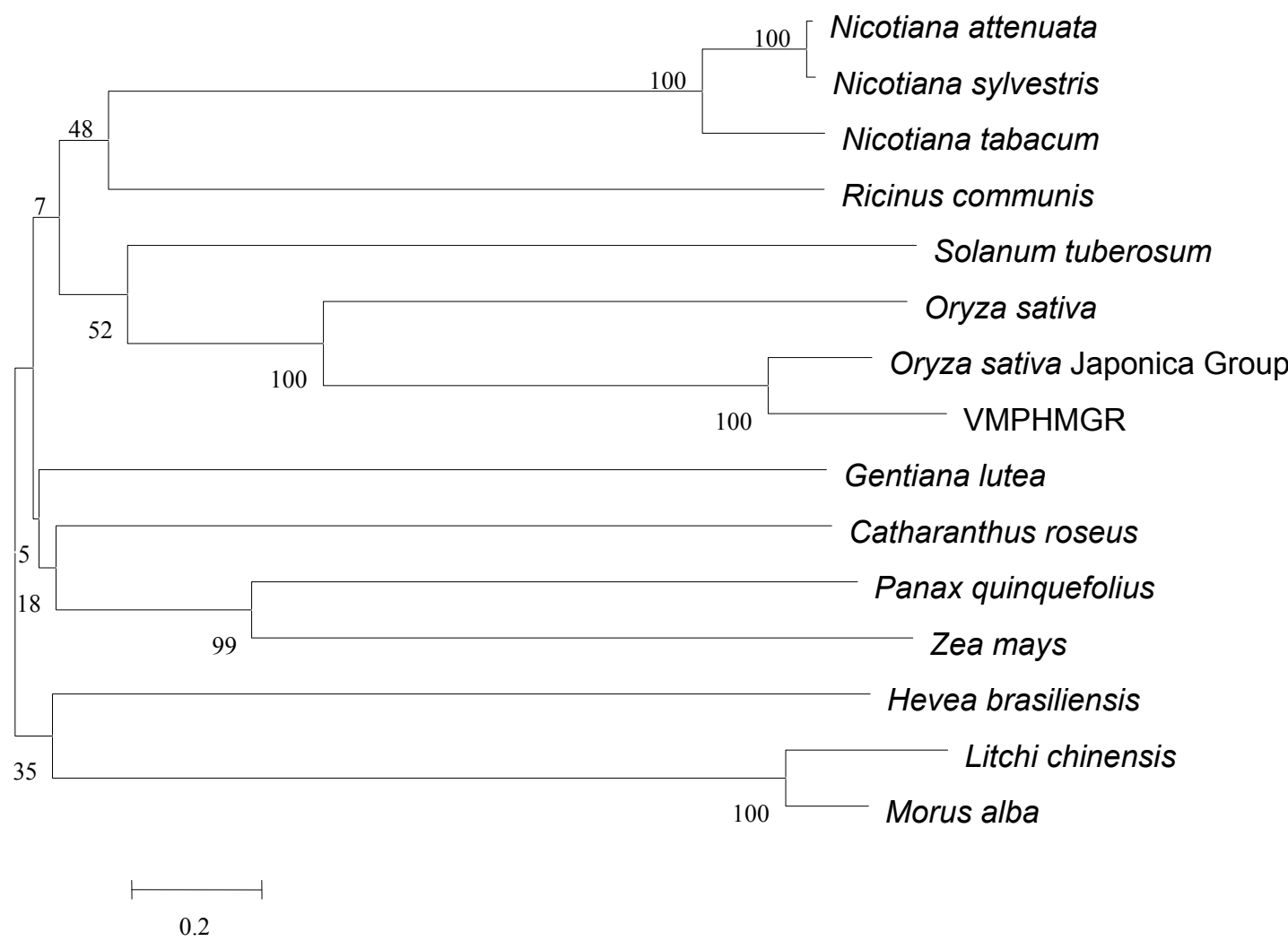

Figure 2. Phylogenetic relationships between $H M G R s$ from various plant sources by MEGA 4.0. The bootstrap neighbour-joining method was used to construct the tree. The phylogenetic tree analysis of $H M G R$ involved those from Zea mays (GenBank accession no. CAA70440), Oryza sativa Japonica group (GenBank accession no. BAD10066.1), Oryza sativa (GenBank accession no. AAD38873.1), Nicotiana tabacum (GenBank accession no. AAB87727.1), Morus alba (GenBank accession no. AAD03789.1), Ricinus communis (GenBank accession no.

EEF52919.1), Solanum tuberosum (GenBank accession no. AAA93498.1), Hevea brasiliensis (GenBank accession no. AAQ63055.1), Nicotiana attenuata (GenBank accession no. AAQ85554.1), Nicotiana sylvestris (GenBank accession no. CAA45181.1), Gentiana lutea (GenBank accession no. BAE92730.1), Litchi chinensis (GenBank accession no. ABF56518.1), Catharanthus roseus (GenBank accession no. AAA33108.1) and Panax quinquefolius (GenBank accession no. ACV65036.1).

\subsection{Expression Analysis of Putative VMPHMGR}

\subsubsection{Developmentally Regulated Expression of Putative VMPHMGR}

In order to understand the molecular profile of $V M P H M G R$, real-time RT-PCR was performed. The putative VMPHMGR has a significantly $(p<0.05)$ high level of expression at the early developmental stage (bud) and lower expressions at both the blooming and full-bloom stages (Figure $3 \mathrm{~A}$ ). The low expressions at the blooming stage imply that HMGR is crucial during early flower development when the flower bud is enlarging and engaging to bloom. These results corresponded well with observations made in other studies (Ha et al., 2003; Narita \& Gruissem, 1989) that HMGR is under transcriptional control during development. Zea mays HMGR also showed a developmentally regulated pattern during seed development and germination (Moore \& Oishi, 1993). Such dominant role of HMGR is noted with it being a key regulatory enzyme in the isoprenoid biosynthesis pathway, crucial in the biosynthesis of mevalonate (MVA) as starting material for the production of 
various isoprenoids (Dudareva et al., 2005). Maldonado-Mendoza et al. (1997) highlighted that HMGR might be supplying the terpenoid intermediates for the formation of indole alkaloids during seeds germination. Jelesko, Jenkins, Rodriguez-Concepcion, and Gruissem (1999) reported that the accumulation of HMGR in tomato was observed only during the early growth phases, and they postulated that HMGR played crucial roles during cell division and growth. Likewise, Narvaez, Canto Canche, Perez, and Madrid (2001) discovered that the highest expression profile of Bixa orellana HMGR was in the immature seed compared to the mature seed.

\subsubsection{Expression of VMPHMGR in Different Floral Parts and Vegetative Tissues}

The VMPHMGR transcript was expressed at significantly $(p<0.05)$ low levels in all tissues compared to the bud (Figure $3 \mathrm{~B}$ ). This may be attributed to the overall low abundance of VMPHMGR which thus diluted its expression level in all parts of the plant. The end product of plant HMGRs (mevalonate) was reported to be beneficial in the physiological responses of plants and in the biosynthesis of carotenoids and sterols for cell membrane maintenance (Pitera et al., 2007). A differential expression profile was also noted for both Arabidopsis thaliana HMGRs (Enjuto et al., 1994). In constrast, Eucommia ulmoides HMGR was found to be a housekeeping gene (Jiang et al., 2006). All the reported studies mentioned above and the differential expression pattern of VMPHMGR imply that VMPHMGR might have discrete functions in regulating the mevalonate pathway.

\subsubsection{Expression of VMPHMGR at Different Time-Points}

The real-time RT-PCR performed on the full-bloom flower using a pair of VMPHMGR-specific primer showed that the expression of VMPHMGR transcript was significantly $(p<0.05)$ highest in the dark with an unusual peaking of approximately 1.3 -fold at 12 a.m., following which slightly lower levels were exhibited throughout the whole morning (Figure 3C) before plunging further at noon. Its expression was low or undetectable in the afternoon and evening (12 noon to 10 p.m.). Enjuto et al. (1994) observed high level of HMGR in Arabidopsis thaliana seedlings placed in the dark. Similar expression profile was also observed for maize $H M G R$ activity during seedlings germination in the dark (Moore \& Oishi, 1993). From the VMPHMGR result, however, it is inconclusive to maintain that the expression level of VMPHMGR is regulated by light since the VMP samples were taken from plants grown under a non-controlled environmental conditions. Besides light, temperature is said to play a significant role in $H M G R$ expression. Low temperature has been shown to increase the expression of HMGR in Picrorhiza kurrooa (Kawoosa et al., 2010). The slightly higher expression levels in the morning (Figure $3 \mathrm{C}$ ) could have been attributed to a slightly cooler temperature. Nevertheless, further experiments with controlled growth conditions, such as placing the plants in a growth chamber, need to be conducted in order to ascertain the expression pattern of VMPHMGR transcript.

\subsection{Cloning and Expression of Recombinant VMPHMGR in E. coli}

Cloning of the coding region of VMPHMGR into pET32(a) for expression in E. coli BL21(DE3)pLysS induced with $0.5 \mathrm{mM}$ IPTG for 4 hours at $30^{\circ} \mathrm{C}$ yielded a protein of $70570 \mathrm{Da}$ (Figures $4 \mathrm{~A}$ and $4 \mathrm{~B}$ ). It was noted that the over-expressed protein was present only in the induced his-tag purified or whole cell lysates of soluble and insoluble fractions. Unfortunately, the $70570 \mathrm{Da}$ band for the whole cell lysates of the induced soluble fractions (Figure 4A, Lane 6) could not be discerned clearly on the SDS-PAGE but was visible in the Western blot (Figure 4B, Lane 3). We attributed this to the higher sensitivity of the Western blot and also the interference of too many cellular proteins in the whole cell lysate sample loaded on the gel. As expected, the whole cell lysate of un-induced soluble fraction did not show the 70570 Da band. Likewise, this band did not appear in the pET32a vector control lane (Figure 4A, Lane 9) implying that the expressed recombinant protein was from the constructed vector itself and not from the $E$. coli expression system.

\subsection{Functional Characterization of a 3-hydroxy-3-methylglutaryl-CoA-reductase of Vanda Mimi Palmer (VMPHMGR)}

The potential products produced from the catalytic activities of VMPHMGR samples comprising of his-tag purified induced protein sample, crude induced and un-induced soluble proteins, and whole cell extract from the host $E$. coli BL21(DE3)pLysS harbouring an empty pET32(a) vector as control, were all subjected to GC-MS. 
(A)

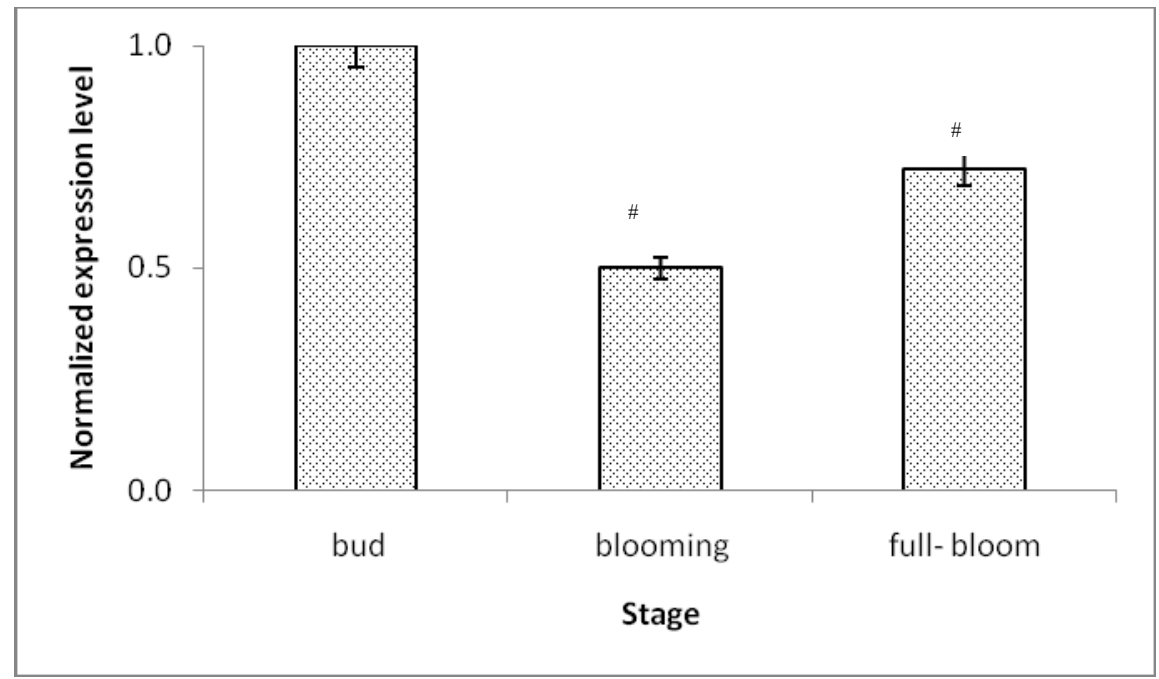

(B)

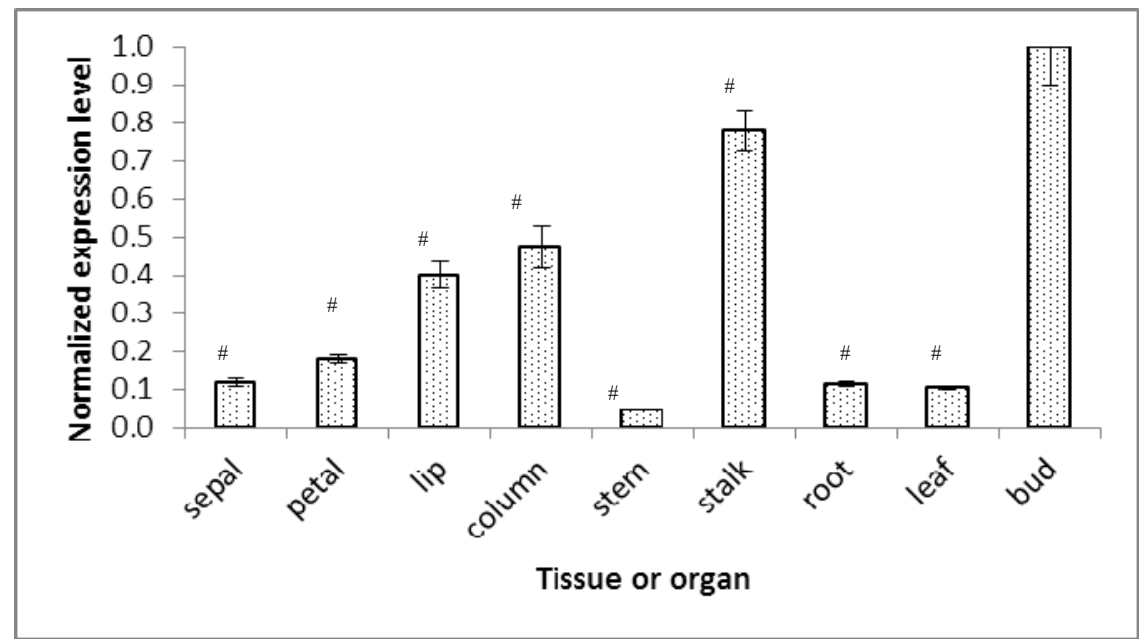

(C)

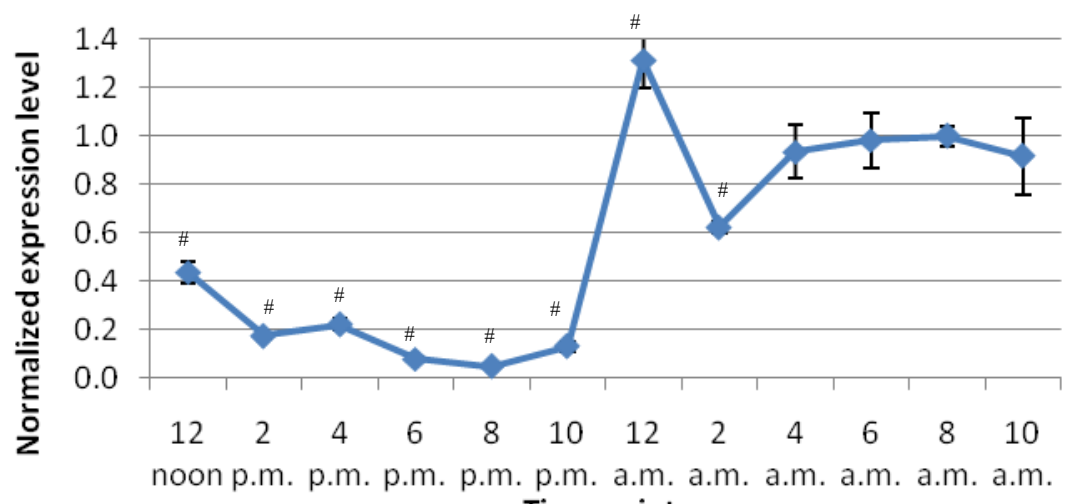

Time points

Figure 3. Expression profile of putative VMPHMGR transcript. Real-time RT-PCR was performed: (A) at different flower developmental stages, (B) in different tissues of VMP and (C) expression in full-bloom flower at different time-points for 2-hour intervals within 24-hour respectively. Error bars represent standard deviations on the normalized ratios. \# indicates significant differences with $p<0.05$ 

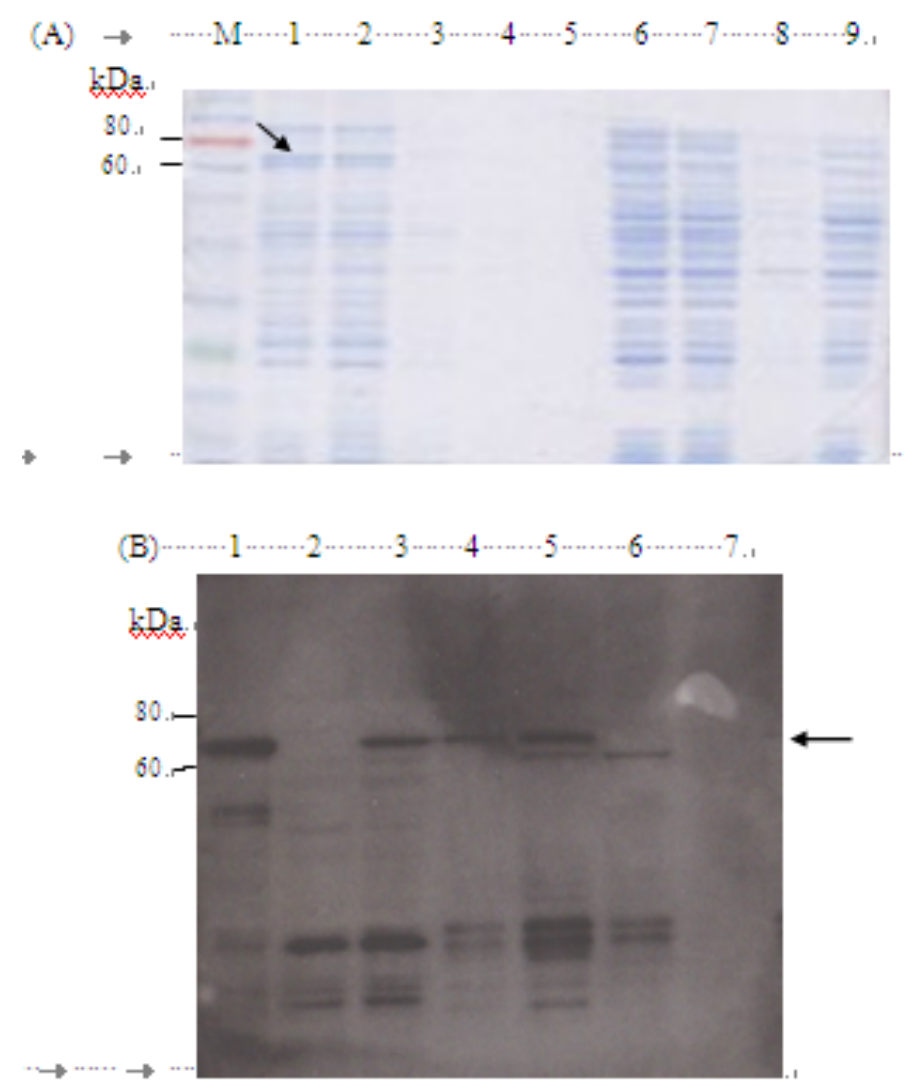

Figure 4. Expression of the putative VMPHMGR in Escherichia coli strain BL21(DE3)pLysS (A) VMPHMGR expression induced with $0.5 \mathrm{mM}$ IPTG at $30^{\circ} \mathrm{C}, 220 \mathrm{rpm}$ for 4 hours. The crude and his-tag purified proteins of each fraction were separated on SDS-PAGE with Coomassie brilliant blue staining. Lane M, molecular weights standard (ColorPlus Prestained Protein Ladder, Broad Range, NEB); Lanes 1-5, his-tag purified induced soluble fraction of VMPHMGR eluted with different imidazole concentrations ( $100 \mathrm{mM}$ to 500 $\mathrm{mM}$ respectively); Lane 6, whole cell lysate of induced soluble VMPHMGR; Lane 7, whole cell lysate of un-induced soluble fraction; Lane 8, whole cell lysate of induced insoluble VMPHMGR; Lane 9, empty pET32(a) vector (without insert). (B) Immunoblot of protein extracts. Lane 1, whole cell lysate of induced insoluble VMPHMGR; Lane 2, whole cell lysate of un-induced soluble fraction; Lane 3, whole cell lysate of induced soluble VMPHMGR; Lanes 4-6, his-tag purified induced soluble fraction of VMPHMGR eluted with different imidazole concentrations ( $300 \mathrm{mM}$ to $100 \mathrm{mM}$ in decreasing order); Lane 7, empty pET32(a) vector (without insert). Arrows indicate over-expressed VMPHMGR of the expected size of 70570 Da.analyses.

Briefly, all the above mentioned protein lysate samples were acidified with $500 \mathrm{mM} \mathrm{HCl}$ to convert the HMG-CoA to mevalonic acid lactone through the reaction of HMGR. The reaction was performed based on the principle that if HMGR is present and functional in the bacterial lysates, it will be able to catalyze the conversion of HMG-CoA to mevalonate, followed by acidification to mevalonate lactone. A crude un-induced soluble VMPHMGR sample was included as a control for detecting potential leakage expression from the constructed plasmid. The end products produced from each of the above enzymatic reactions were subjected to GC-MS analysis.

The GC-MS single ion chromatogram showed two components from the above reaction (Figure 5 A). Dehydromevalonic lactone and pantolactone were detected at the retention times of 11.50 minutes and 13.20 minutes, respectively. By comparing the mass spectra of each component produced [Figures $5 \mathrm{~B}$ (i) and $5 \mathrm{C}$ (i)] with the values at the NIST 2008 and Wiley mass spectral libraries, dehydromevalonic lactone was identified as the main spectrum fragments of $\mathrm{m} / \mathrm{z} 54,67,82$ and 112 while pantolactone as the main spectrum fragments of $\mathrm{m} / \mathrm{z}$ 53, 58, 71, 85, 103, 115 and 131 [Figure $5 \mathrm{~B}$ (ii) and C(ii), respectively]. This shows that the recombinant VMPHMGR was functional and active in catalyzing the conversion of HMG-CoA to derivatives of mevalonate lactone. 
Lactone was not detected from the crude un-induced soluble protein sample [Figure $5 \mathrm{D}$ (i) and E (i)] as seen from the comparison made between the mass spectra of each component produced with the values at the NIST 2008 and Wiley libraries [Figures 5 D (ii) and E (ii)], indicating no leakage expression from the construct as the BL21(DE3)pLysS system avoids un-induced expression. In addition, no lactone was detected in the control lysate prepared from the bacterial cells harbouring the empty plasmid (without the VMPHMGR insert).

(A)

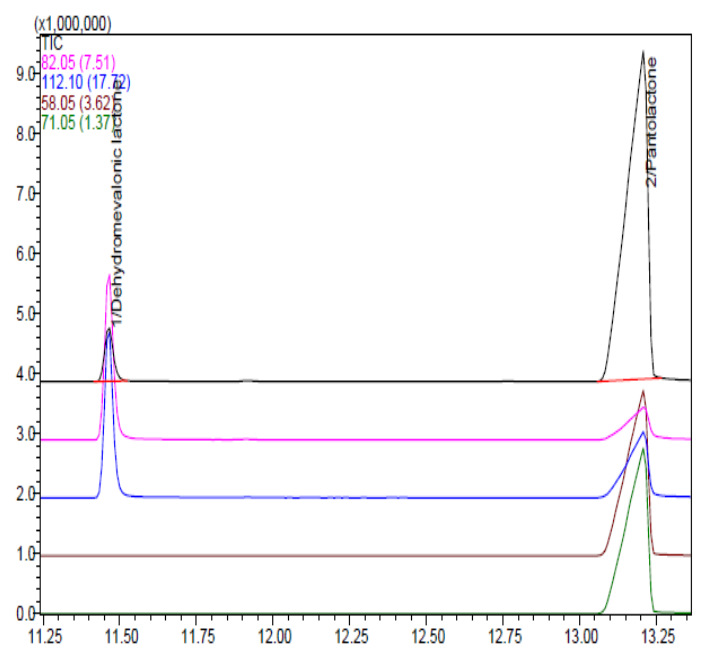

(C)(i) Sample

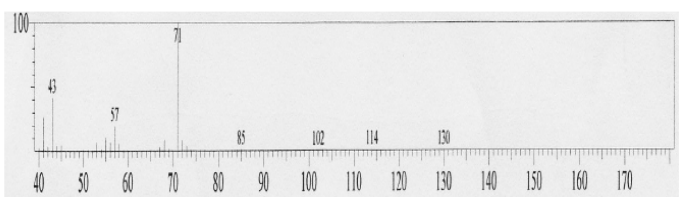

(ii) Libraries

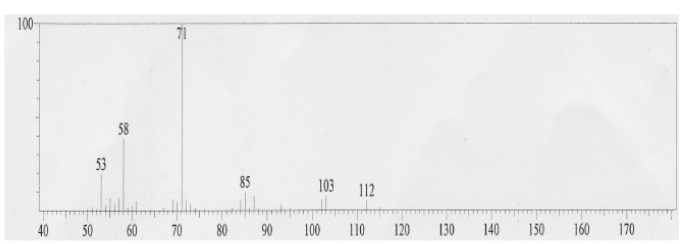

(B)(i) Sample

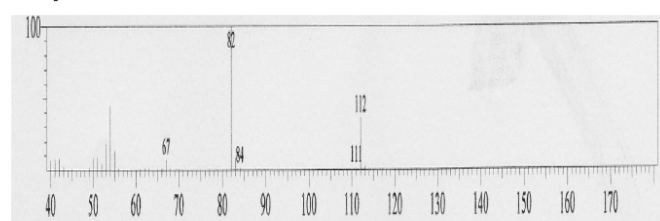

(ii) Librarie

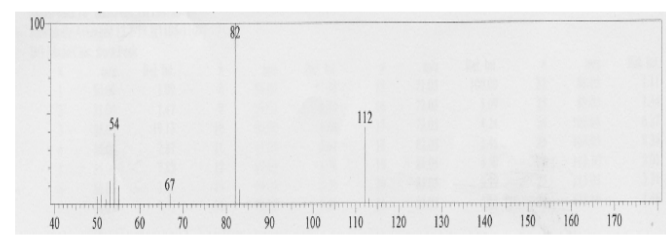

(D)(i) Sample (control)

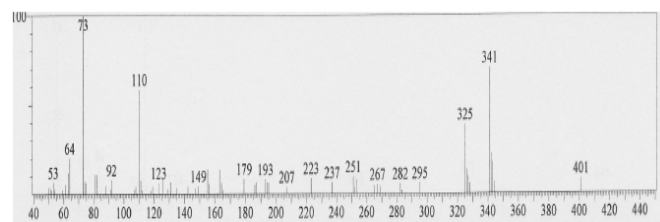

(ii) Libraries

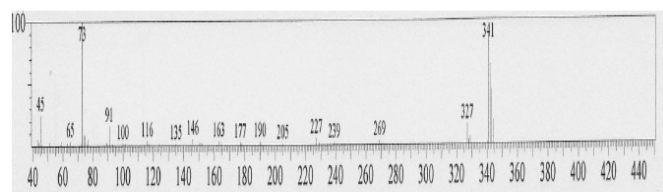

(E)(i) Sample (control)

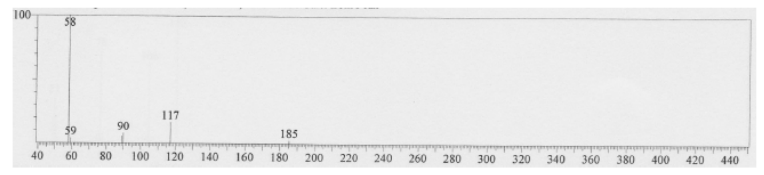

(ii) Libraries

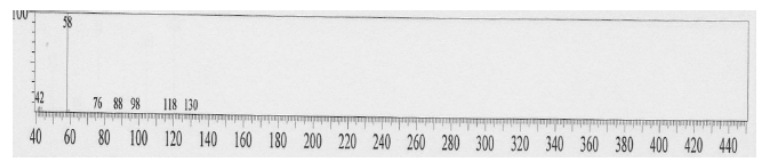

Figure 5. GC-MS analyses of products formed from the enzymatic activity of VMPHMGR. (A) Single ion chromatogram formed from the crude induced soluble VMPHMGR. Peaks with different colours indicate the 
mass spectra of corresponding products at respective retention times. (B) (i) and (C) (i) are mass spectra of corresponding products produced from crude induced soluble VMPHMGR while (B) (ii) and (C) (ii) are the highest hit with NIST 2008 and Wiley libraries. (D) (i) and (E) (i) are mass spectra of corresponding products produced from crude un-induced soluble VMPHMGR (control) while (D) (ii) and (E) (ii) showed the chromatograms of the highest match with NIST 2008 and Wiley libraries.

\section{Conclusion}

This is the first report on the over-expression and biochemical characterization of a truncated recombinant HMGR protein, from a vandaceous orchid, produced in the E. coli system. VMPHMGR was functionally expressed in E. coli and GC-MS analysis showed its catalytic activities resulted in the production of dehydromevalonic lactone and pantolactone. Such successful molecular and biochemical results would lend a better understanding of fragrance production in vandaceous orchids. Current work in our laboratory is focusing on increasing the solubility and purity of the recombinant protein for use in a wider range of biochemical and catalytic studies of this fragrance-related enzyme.

\section{Acknowledgements}

We would like to thank the Ministry of Higher Education Malaysia and Universiti Putra Malaysia for financial support through grants, Fundamental Research Grant Scheme (01-11-08-6470FR and 02-12-10-1002FR) and Research University Grant Scheme (05-04-08-0551RU), respectively. We thank Ms Adelene Song for helpful comments and advices in the functional enzymatic analysis.

\section{References}

Bendsten, J. D., Nielsen, H., Heijne, G., \& Brunak, S. (2004). Improved prediction of signal peptides-SignalP 3.0. Journal of Molecular Biology, 340(4), 783-795. http://dx.doi.org/10.1016/j.jmb.2004.05.028

Chan, W. S., Janna, O. A., Namasivayam, P., \& Maziah, M. (2009). Molecular characterization of a new 1-deoxy-D-xylulose 5-phosphate reductoisomerase (DXR) transcript from Vanda Mimi Palmer. Scientia Horticulturae, 121(3), 378-382. http://dx.doi.org/10.1016/j.scienta.2009.02.015

Chan, W. S., Janna, O. A., \& Namasivayam, P. (2011). Isolation, cloning and characterization of fragrance-related transcripts from Vanda Mimi Palmer. Scientia Horticulturae, 127(3), 388-397. http://dx.doi.org/10.1016/j.scienta.2010.09.024

Chappell, J., Wolf, F., Proulx, J., Ceullar, R., \& Saunders, C. (1995). Is the reaction catalyzed by 3-hydroxy-3-methylglutaryl coenzyme A reductase a rate-limiting step for isoprenoid biosynthesis in plants? Plant Physiology, 109 (4), 1337-1343.

Cheng, A.-X., Lou, Y. G., Mao, Y. B., Lu, S., Wang, L. J., \& Chen, X. Y. (2007). Plant terpenoids: biosynthesis and ecological functions. Journal of Integrative Plant Biology, 49(2), 179-186. http://dx.doi.org/10.1111/j.1744-7909.2007.00395.x

Donald, K. A., Hampton, R. Y., \& Fritz, I. B. (1997). Effects of overproduction of the catalytic domain of 3-hydroxy-3-methylglutaryl coenzyme A reductase on squalene synthesis in Saccharomyces cerevisiae. Applied and Environmental Microbiology, 6 (9), 3341-3344.

Dudavera, N., Martin, D., Kish, C. M., Kolosova, N., Gorenstein, N., Faldt, J., ... Bohlmann, J. (2003). (E)- $\beta$-Ocimene and myrcene synthase genes of floral scent biosynthesis in snapdragon: Function and expression of three synthase genes of a new terpene synthase subfamily. The Plant Cell, 15, 1227-1241. http://dx.doi.org/10.1105/tpc.011015

Dudareva, N., Andersson, S., Orlova, I., Gatto, N., Reichelt, M., Rhodes, D., Boland, W., \& Gershenzon, J. (2005). The nonmevalonate pathway supports both monoterpene and sesquiterpene formation in snapdragon flowers. PNAS, 102(3), 933-938. http://dx.doi.org/10.1073/pnas.0407360102

Enjuto, M., Balcells, L., Campos, N., Caelles, C., Arro, M., \& Boronat, A. (1994). Arabidopsis thaliana contains two differentially expressed 3-hydroxy-3-methylglutaryl-CoA reductase genes, which encode microsomal forms of the enzyme. Plant Biology, 91 (3), 927-931.

Friesen, J. A., \& Rodwell, V. W. (2004). Protein family review: the 3-hydroxy-3-methylglutaryl-coenzyme A (HMG-CoA) reductases. Genome Biology, 5(11), 248-254. http://dx.doi.org/10.1186/gb-2004-5-11-248

Geourjon, C., \& Deleage, G. (1995). SOPMA: significant improvement in protein secondary structure prediction by consensus prediction from multiple alignments. CABIOS, 11(6), 681-684. 
Goff, S., \& Klee, H. (2006). Plant volatile compounds: sensory cues for health and nutritional value? Science, 311(5762), 815-819. http://dx.doi.org/10.1126/science.1112614

Guterman, I., Shalit, M., Menda, N., Piestun, D., Dafny, Y., Shalev, G., ... Weiss, D. (2002). Rose scent: genomics approach to discovering novel floral fragrance-related genes. The Plant Cell, 14(10), 2325-2338. http://dx.doi.org/10.1105/tpc.005207

Ha, S. H., Lee, S. W., Kim, Y. M., \& Hwang, Y. S. (2001). Molecular characterization of Hmg2 gene encoding a 3-hydroxy-3-methylglutaryl-CoA reductase in rice. Molecules and Cells, 11(3), 295-302.

Ha, S. H., Kim, J. B., Hwang, Y. S., \& Lee, S. W. (2003). Molecular characterization of three 3-hydroxy-3-methylglutaryl-CoA reductase genes including pathogen-induced Hmg2 from pepper (Capsicum annuum). Biochimica et Biophysica Acta (BBA), 1625(3), 253-260. http://dx.doi.org/10.1016/S0167-4781(02)00624-3

Istvan, E. S. (2001). Bacterial and mammalian HMG-CoA reductases: related enzymes with distinct architectures. Current Opinion in Structural Biology, 11(6), 746-751. http://dx.doi.org/10.1016/S0959-440X(01)00276-7

Jackson, B. E., Hart-Wells, E. A., \& Matsuda, S. P. T. (2003). Metabolic engineering to produce sesquiterpenes in yeast. Organic Letters, 5(10), 1629-1632.

Jelesko, J., Jenkins, S., Rodriguez-Concepcion, M., \& Gruissem, W. (1999). Regulation of tomato HMG1 during cell proliferation and growth. Planta, 208(3), 310-318. http://dx.doi.org/10.1007/s004250050564

Jiang, J. H., Kai, G. Y., Cao, X. Y., Chen, F. M., He, D. N., \& Liu, Q. (2006). Molecular cloning of a HMG-CoA reductase gene from Eucommia ulmoides oliver. Bioscience Reports, 26(2), 171-181. http://dx.doi.org/10.1007/s10540-006-9010-3

Karlen, Y., McNair, A., Perseguers, S., Mazza, C., \& Mermod, N. (2007). Statistical significance of quantitative PCR. BMC Bioinformatics, 8, 131-147. http://dx.doi.org/10.1186/1471-2105-8-131

Kawoosa, T., Singh, H., Kumar, A., Sharma, S. K., Devi, K., Dutt, S., ... Kumar, S. (2010). Light and temperature regulated terpene biosynthesis: hepatoprotective monoterpene picroside accumulation in Picrorhiza kurrooa. Functional and Integrative Genomics, 10, 393-404. http://dx.doi.org/10.1007/s10142-009-0152-9

Kevei, Z., Lougnon, G., Mergaert, P., Horvath, G. V., Kereszt, A., Jayaraman, D., ... Ane, J. M. (2007). 3-hydroxy-3-methylglutaryl coenzyme A reductase I interacts with NORK and is crucial for nodulation in Medicago truncatula. The Plant Cell, 19(12), 3974-3989. http://dx.doi.org/10.1105/tpc.107.053975

Knudsen, J., \& Gershenzon, J. (2006). The chemical diversity of floral scent. In: Dudareva N, Pichersky E. (eds) Biology of floral scent. CRC Press, Florida, pp. 27-52.

Maldonado-Mendoza, I. E., Vincent, R. M., \& Nessler, C. L. (1997). Molecular characterization of three differentially expressed members of the Camptotheca acuminate 3-hydroxy-3-methylglutaryl CoA reductase (HMGR) gene family. Plant Molecular Biology, 34(5), 781-790. http://dx.doi.org/10.1023/A:1005866813347

Mohd Hairul, A. R. (2010). Chemical composition of floral volatiles and expression of scent-related genes in Vanda Mimi Palmer. Master dissertation, Universiti Putra Malaysia, Selangor Darul Ehsan, Malaysia.

Mohd Hairul, A. R., Namasivayam, P., Gwendoline, E. C. L., \& Janna O. A. (2010). Terpenoid, benzenoid and phenylpropanoid compounds in the floral scent of Vanda Mimi Palmer. Journal of Plant Biology, 53(5), 358-366. http://dx.doi.org/10.1007/s12374-010-9123-x

Moore, K. B., \& Oishi, K. K. (1993). Characterization of 3-hydroxy-3-methylglutaryl coenzyme A reductase activity during maize seed development, germination and seedling emergence. Plant Physiology, 101(2), 485-491.

Narita, J. O., \& Gruissem, W. (1989). Tomato hydroxymethylglutaryl-CoA reductase is required early in fruit development but not during ripening. The Plant Cell, 1(2), 181-190.

Narvaez, J. A., Canto, C. B. B., Perez, P. F., \& Madrid, R. R. (2001). Differential expression of 3-hydroxy-3-methylglutaryl-CoA reductase (HMGR) during flower and fruit development of Bixa orellana. Journal of Plant Physiology, 158(11), 1471-1477. http://dx.doi.org/10.1078/0176-1617-00614

Nelson, A. J., Doerner, P. W., Zhu, Q., \& Lamb, C. J. (1994). Isolation of a monocot 3-hydroxy-3-methylglutaryl coenzyme A reductase gene that is elicitor-inducible. Plant Molecular Biology, 25, 401-412. 
Pichersky, E., Noel, J., \& Durareva N. (2006). Biosynthesis of plant volatiles: nature's diversity and ingenuity. Science, 311(5762), 808-811. http://dx.doi.org/10.1126/science.1118510

Pitera, D., Paddon, C., Newman, J., \& Keasling, J. (2007). Balancing a heterologous mevalonate pathway for improved isoprenoid production in Escherichia coli. Metabolic Engineering, 9(2), 193-207. http://dx.doi.org/10.1016/j.ymben.2006.11.002

Rohmer, M., Knani, M., Simonin, P., Sutter, B., \& Sahm, H. (1993). Isoprenoid biosynthesis in bacteria: a novel pathway for the early steps leading to isopentenyl diphosphate. Biochemistry Journal, 295(2), 517-524.

Shang, C. H., Zhu, F., Li, N., Ou-Yang, X., Shi, L., Zhao, M. W., \& Li, Y. X. (2008). Cloning and characterization of a gene encoding HMG-CoA reductase from Ganoderma lucidum and its functional identification in yeast. Bioscience, Biotechnology and Biochemistry, 72(5), 1333-1339. http://dx.doi.org/10.1271/bbb.80011

Tada, M., Tsubouchi, M., Matsuo, K., Takimoto, H., Kimura, Y., \& Takagi, S. (1990). Mechanism of photoregulated carotenogenesis in Rhodotorula minuta VIII. Effect of mevinolin on photoinduced carotenogenesis. Plant Cell Physiology, 31(3), 319-323.

Teh, S. L, Chan, W. S., Janna, O. A., \& Namasivayam, P. (2011). Development of expressed sequence tag resources for Vanda Mimi Palmer and data mining for EST-SSR. Molecular Biology Report, 38(6), 3903-3909. http://dx.doi.org/10.1007/s11033-010-0506-3

Vandesompele, J., De Preter, K., Pattyn, F., Poppe, B., Van Roy, N., De Paepe, A., \& Speleman, F. (2002). Accurate normalization of real-time RT-PCR data by geometric averaging of multiple internal control genes. Genome Biology, 3(7), 1-12. http://dx.doi.org/10.1186/gb-2002-3-7-research0034

Wang, G. Y., \& Keasling, J. D. (2002). Amplification of HMG-CoA reductase production enhances carotenoid accumulation in Neurospora crassa. Metabolic Engineering, 4(3), 193-201. http://dx.doi.org/10.1006/mben.2002.0225

Wang, Y. C., Guo, B. H., Zhang, F., Yao, H. Y., Miao, Z. Q., \& Tang, K. X. (2007). Molecular cloning and functional analysis of the gene encoding 3-hydroxy-3-methylglutaryl coenzyme A reductase from hazel (Corylus avellana L. Gasaway). Journal of Biochemistry and Molecular Biology, 40(6), 861-869.

Yu, H., \& Goh, C. J. (2000). Identification and characterization of three orchid MADS Box genes of the AP1/APL9 subfamily during floral transition. Plant Physiology, 123, 1325-1336. http://dx.doi.org/10.1104/pp.123.4.1325 